\title{
Recombinant Lz-8 from Ganoderma lucidum induces endoplasmic reticulum stress-mediated autophagic cell death in SGC-7901 human gastric cancer cells
}

\author{
CHONGYANG LIANG* ${ }^{*}$ HONGRUI LI*, HUI ZHOU, SHUQIN ZHANG, ZHIYI LIU, QIULI ZHOU and FEI SUN
}

Institute of Frontier Medical Science of Jilin University, Changchun 130021, P.R. China

Received September 17, 2011; Accepted November 15, 2011

DOI: 10.3892/or.2011.1593

\begin{abstract}
In Asia, the mushroom of the fungus Ganoderma lucidum has been widely used as a traditional medicine for the past two millennia. The aim of this study was to investigate the anticancer activity of recombinant Lz-8 (rLz-8), a protein belonging to a family of fungal immunomodulatory proteins. We report that rLz-8 induces endoplasmic reticulum (ER) stressmediated autophagic cell death in the human gastric cancer cell line SGC-7901. Our results show that rLz-8 induces autophagic cell death by aggregating in the ER, triggering ER stress and the ATF4-CHOP pathway. A foreign protein, in the ER rLz-8 causes the activation of the ubiquitine/proteasome ER-associated degradation (ERAD) system. The autophagic arm of this system is then overstimulated by an excessive abundance of $\mathrm{rLz}-8$ and causes the cell's death through an over-autophagic response. We also found that caspase inhibitors do not prevent rLz-8-induced cell death, and therefore the autophagic response induced by rLz-8 is independent of caspase activation.
\end{abstract}

\section{Introduction}

Proteins must be folded into proper conformations in order to carry out their cellular functions (1). The endoplasmic reticulum (ER) is the site of synthesis and folding of proteins destined for either secretion or locations in the cell membrane, Golgi apparatus, lysosomes and elsewhere (2). However, when protein folding in the ER is impaired due to various physiological and pathological conditions, unfolded or misfolded proteins can threaten cell survival. The unfolded protein response (UPR) of the cell stresses the ER and triggers an ER quality-control

Correspondence to: Dr Fei Sun, Institute of Frontier Medical Science of Jilin University, 1163 Xinmin Street, Changchun 130021, P.R. China

E-mail: s.fei1958@gmail.com

*Shared first co-authorship

Key words: Ganoderma lucidum, rLz-8, autophagy, endoplasmic reticulum, ER-associated degradation system that recognizes and removes nascent proteins that fail to fold or assemble properly $(3,4)$.

The UPR system is composed of two ER-associated degradation (ERAD) systems, the ubiquitin/proteasome and the autophagy/lysosome (5). Fujita et al (5) used dystrophyassociated fer-1-like protein (dysferlin) degradation to investigate two ERAD models and found that when misfolded dysferlin aggregated on the ER membrane excessively, the cell chose the autophagy/lysosome ERAD system rather than the ubiquitin/proteasome (6,7). It is known that autophagy is a self-digestion process that degrades intracellular structures in response to stresses, whose purpose is cell survival. However, if autophagy was prolonged, it leads to cell death (8). Therefore, the autophagy/lysosome ERAD system may open a door for purposively causing cell death.

In recent years, the relationship between the intracellular aggregation of unfolded or misfolded proteins and ER stress has been intensively examined (9). However, there are few reports in the cell biological literature regarding ER stress and autophagy induced by a heterologous protein. In this study, we found that recombinant Lz-8 (rLz-8), a protein from the fruiting body of the bracket fungus Ganoderma lucidum, aggregated on the ER of human gastric cancer SGC-7901 cells. Accumulation of rLz-8 induced ER stress and autophagy, leading to massive death of cells, independent of caspase activity. The aims of this study were to determine how cells deal with a heterologous protein such as rLz-8 that accumulates on the ER, and the role of ER stress and autophagy in triggering cell death.

Ganoderma lucidum, a popular medicinal mushroom, has been widely used in traditional Chinese medicine in many Asian countries during the past two millennia. It has been reported to be effective in modulating immune functions, inhibiting tumor cell growth and allergies (10) and in the treatment of chronic hepatitis, hypertension, and hyperglycemia (11). G. lucidum polysaccharide and triterperoid were the major bioactive substances until immunomodulatory proteins, Lz-8, was isolated and purified from the mycelia of G. lucidum in 1989 (12-14). Previously, we demonstrated the crystal structure of Lz-8 which was a noncovalently linked homodimer with an apparent molecular weight of $24 \mathrm{kDa}$. Each monomer consists of 110 aa residues with an acetylated $\mathrm{N}$ terminus and a molecular mass of $12 \mathrm{kDa}(15)$. Liao et al also reported that reFIP-gts, another immunomodulatory protein from the closely related 
Ganoderma tsugae, inhibited the growth of A549 cancer cells significantly and selectively (16). However, until now there have been no studies demonstrating how Lz-8 induces cell death and the mechanisms involved in this process.

Herein we report for the first time that an excessive heterologous protein aggregation of rLz-8 from Ganoderma lucidum on the ER of human cancer cells induces autophagy-dependent cell death, not a caspase-dependent cell death or apoptosis, and provides a novel strategy for cancer treatment.

\section{Materials and methods}

Recombinant plasmid construction and Pichia pastoris transformation. The total DNA of G. lucidum was extracted as described by Al-Samarrai and Schmid (17). The rLz-8 gene was amplified from the total DNA sample by PCR, and SnaBI and EcoRI restriction sites were designed for flanking the PCR product at the 5'- and 3'- termini, respectively. To build the expression plasmid, the PCR fragment was cloned into the SnaBI/EcoRI site of pPIC9K (Invitrogen, CA, USA) and transformed into Pichia pastoris Gs115 (Mut'; Invitrogen).

Media and culture conditions for rLz-8 expression. The $P$. pastoris transformants were cultured in a $100 \mathrm{ml}$ flask containing $1000 \mathrm{ml}$ buffered minimal glycerol-rich yeast (BMGY) medium, supplemented with $1 \%$ (v/v) glycerol as a carbon source and $200 \mu \mathrm{g} / \mathrm{ml} \mathrm{G} 418$ (Geneticin) as a selection pressure. Cells were grown at $28^{\circ} \mathrm{C}$ and shaken at $300 \mathrm{rpm}$ until an $\mathrm{OD}_{600}$ value reached 15 making a 10 -fold dilution. The cells were then harvested by centrifugation at $3000 \mathrm{x} \mathrm{g}$ and $4^{\circ} \mathrm{C}$ for $5 \mathrm{~min}$. BMGY was replaced by buffered minimal methanol-rich yeast (BMMY) medium containing $0.5 \%(\mathrm{v} / \mathrm{v})$ methanol. Methanol was used to induce the AOXI promoter. BMGY and BMMY media were also prepared according to the manufacturer's instructions (Invitrogen). After $48 \mathrm{~h}$ of induction, the supernatant was collected by centrifugation at $4^{\circ} \mathrm{C}$ and $12,000 \times \mathrm{g}$ for $20 \mathrm{~min}$. $\mathrm{rLz}-8$ protein was purified by a nickel affinity column Ni-sepharose (GE Lifescience, USA) and eluted by a gradient of 30-100 mM imidazol.

Cell culture and materials. Cells of the human gastric cancer cell line SGC7901 were obtained from the Institute of Biochemistry and Cell Biology (Shanghai, China). Cells were grown in Dulbecco's modified Eagle's medium (DMEM; Invitrogen) supplemented with $2 \mathrm{mM}$ L-glutamine, $100 \mathrm{U} / \mathrm{ml}$ penicillin, $100 \mu \mathrm{g} / \mathrm{ml}$ streptomycin, and $10 \%$ fetal bovine serum (FBS) at $37^{\circ} \mathrm{C}$ in a humidified atmosphere of $95 \%$ air and $5 \% \mathrm{CO}_{2}$. Anti-ATF4 antibody was purchased from Proteintech Group (PTG, USA). Anti-CHOP, Anti-LC3, Anti-GAPDH antibodies and secondary antibody were purchased from Santa Cruz Biotechnology (Santa Cruz, Canada). Tm, TG and 3-MA were obtained from Sigma-Aldrich (USA).

Electron microscopy. SGC7901 cells were double fixed in PBS-buffered glutaraldehyde (2.5\%) and osmium tetroxide (1\%), dehydrated, and embedded using the EMBed-812 kit (EMS, USA) according to the manufacturer's instructions. Ultrathin sections $(70 \mathrm{~nm})$ were made and double stained with uranyl acetate and lead citrate, and viewed in a transmission electron microscope (Hitachi H-7500, Japan).
Western blotting. Cells were washed with PBS and lysed in Lysis-M Reagent supplemented with complete mini-protease inhibitor cocktail tablets (Roche, Indianapolis, IN, USA). The lysates were then incubated for $5 \mathrm{~min}$ at room temperature with gentle shaking, and centrifuged at $14,000 \mathrm{x} \mathrm{g}$ for $10 \mathrm{~min}$. Equal amounts of protein were subjected to $12 \%$ sodium dodecyl sulfate polyacrylamide gel electrophoresis and then transferred to polyvinylidene difluoride membranes (Millipore, USA). The membranes after blocking in 5\% skimmed milk powder in TBS were incubated with each primary antibody followed by a peroxidase conjugated anti-rabbit secondary antibody. The corresponding bands were detected using an ECL Advance kit (GE Biosciences, USA).

Real-time quantitative PCR. The total RNA was isolated using Trizol (Invitrogen) from cells after treatment with or without rLz-8 for the indicated times. cDNA was synthesized using a reverse transcription system (Takara, Japan). The real-time PCR was performed with the use of LineGene 3320 Bioanalyzer (Bioer, China) following the manufacturer's instructions. SYBR Green I kit (Takara) was used. PCR conditions were $95^{\circ} \mathrm{C}$ for $30 \mathrm{sec}$ followed by 40 cycles of $95^{\circ} \mathrm{C}$ for $5 \mathrm{sec}$ and $60^{\circ} \mathrm{C}$ for $20 \mathrm{sec}$. Bioer 3320 software was used to analyze the standards and to carry out the quantification. The relative quantity of each mRNA was normalized to the relative quantity of GAPDH mRNA. ATF4 sense primer: 5' -TCTCC AGCGACAAGGCT AAG-3'; ATF4 anti-sense primer: 5' -TACCCAACAGGGCATC CAAG-3'; CHOP sense primer: 5'-GAACCAGGAAACGGAA ACAG-3'; CHOP anti-sense primer: 5' -CATTCACCATTCGG TCAATCA-3'; LC3 sense primer: 5'-AGCAGCATCCAACC AAAATC-3'; LC3 anti-sense primer: 5'-CTGTGTCCGTTCA CCAACAG-3'; GAPDN sense primer: 5'-GCACCGTCAAGG CTGAGAAC-3'; GAPDH anti-sense primer: 5'-ATGGTGGTG AAGACGCCAGT-3'.

Cell proliferation assays. The inhibition of cell proliferation and viability in SGC7901 cells was determined using the WST-1 (Roche) assay. Cells were placed at 8,000 per well in 96-well plates in their respective growth medium with FBS reduced to $5 \%$. The cells were allowed to grow for $24 \mathrm{~h}$ and then treated with different drugs. After $24 \mathrm{~h}$, WST-1 reagent was added to the plates according to manufacturer's protocol, and absorbance was read at $450 \mathrm{~nm}$ with an ELISA reader (Tecan).

Confocal microscopy. ERtracker, mitotracker and lysotracker (Invitrogen) was used for the assessment of cellular lysosomal content, according to the manufacturer's instructions. Briefly, cells were cultured on 24-well plates and treated with $\mathrm{rLz}-8$ - fluorescein isothiocyanate (FITC) or $\mathrm{rLz}-8(5 \mu \mathrm{g} / \mathrm{ml})$. After several time courses, cells were incubated with $1 \mu \mathrm{M}$ ER tracker, $300 \mathrm{nM}$ mitotracker, or $75 \mathrm{nM}$ Lysotracker for $45 \mathrm{~min}$ at $37^{\circ} \mathrm{C}$ in $5 \% \mathrm{CO}_{2}$, and nuclear stained with $1 \mu \mathrm{g} / \mathrm{ml}$ Hoechst33342 (Sigma, USA) for $15 \mathrm{~min}$. Cell were washed thrice with fresh medium and were visualized using an Olympus laser scanning confocal fluorescence microscope fitted with the appropriate filters. The results of Lysotracker fluorescence were selected, five fields of view (512x512 pixel) per section, and fluorescence images were analyzed using ImageJ software (NIH). 
Measurement of caspase activity. Caspase-3 activity in SGC7901 cells was measured with a NucView 488 Caspase-3 Assay Kit for live cells according to the manufacturer's instructions (Biotium, Hayward, CA, USA). Briefly, the cells were grown in 24-well plates at a density of $1 \times 10^{5}$ cells per well. After $\mathrm{rLz}-8$ treatment for $24 \mathrm{~h}$, cells were incubated with $3 \mu \mathrm{m}$ NucView 488 Caspase-3 substrate in medium for $30 \mathrm{~min}$. Cells (200/group) were visualized using a fluorescence microscope fitted with the appropriate filters. For the inhibition of caspases, cells were grown in 96-well plates. After $24 \mathrm{~h}$, the cells were pre-incubated with $50 \mu \mathrm{M}$ z-VAD-fmk (a pan-caspase inhibitor) for $30 \mathrm{~min}$ and then treated with $\mathrm{rLz}-8$ for $48 \mathrm{~h}$. The results were measured with the WST-1 assay.

Cell cycle analysis. Cells were placed into 6-well plates at a density of $5 \times 10^{5}$ cells per well and incubated with media or $\mathrm{rLz}-8$ at $5 \mu \mathrm{g} / \mathrm{ml}$ for the indicated times. Cells were harvested by centrifugation at appropriate times and fixed with $70 \%$ ethanol at $4^{\circ} \mathrm{C}$ overnight. After fixation, cells were washed by centrifugation with PBS and resuspended in $1 \mathrm{ml}$ PI staining buffer $(0.1 \%$ Triton X-100, $100 \mu \mathrm{g} / \mathrm{ml}$ RNase A, $50 \mu \mathrm{g} / \mathrm{ml}$ PI in PBS) at $37^{\circ} \mathrm{C}$ for $30 \mathrm{~min}$. The cell cycle phase was analyzed using a flow cytometer (Becton Dickinson, USA) with Cellquest software.

Assessment of apoptosis. The effects of rLz-8 on nuclear morphology in cells were examined by staining with Hoechst

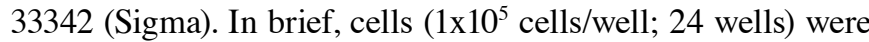
incubated with $\mathrm{rLz}-8(5 \mu \mathrm{g} / \mathrm{ml})$ in the growth medium for $24 \mathrm{~h}$ at $37^{\circ} \mathrm{C}$. The cells were stained with Hoechst $33342(1 \mu \mathrm{g} / \mathrm{ml})$ for 15 min and washed twice with PBS. The images were observed under a fluorescence microscope (Olympus X71, Japan).

Evaluation of apoptotic cell death was performed with the Annexin V-FITC Apoptosis Detection kit (Bipec Biopharma, USA) according to the manufacturer's recommendations. In brief, after the treatment, cells were washed twice with cold PBS and collected by centrifugation. The pellet was resuspended in $1 \mathrm{X}$ binding buffer and stained with FITC-labeled Annexin V $(5 \mu \mathrm{l})$ for $15 \mathrm{~min}$ at $4-8^{\circ} \mathrm{C}$ in the dark. PI $(10 \mu \mathrm{l})$ was then added for $5 \mathrm{~min}$ at $4-8^{\circ} \mathrm{C}$ in the dark. Cells were analyzed using a flow cytometer (Becton Dickinson) with Cellquest software.

Mitochondrial membrane potential. Mitochondrial membrane potential was examined by JC-1, according to the manufacturer's instructions. Briefly, cells were placed on 6-well plates and treated with rLz-8 $(5 \mu \mathrm{g} / \mathrm{ml})$. After $24 \mathrm{~h}$, cells were incubated with $10 \mu \mathrm{g} / \mathrm{ml} \mathrm{JC}-1$ for $30 \mathrm{~min}$ at $37^{\circ} \mathrm{C}$ in $5 \% \mathrm{CO}_{2}$ and then analyzed using a flow cytometer.

Small interfering RNA (siRNA). The ATF4 siRNA or CHOP siRNA duplexes used in this study were purchased from Santa Cruz Biotechnology. SGC7901 cells, plated 1 day earlier on 6-well culture dishes to achieve 50-60\% confluence, were transfected with siRNAs ( $80 \mathrm{pM})$ by using $6 \mu$ l of Lipofectmine 2000 (Invitrogen). After $24 \mathrm{~h}$ of transfection, cells were treated with rLz-8 for up to $24 \mathrm{~h}$.

Statistical analyses. Statistical analysis was conducted using Student's t-test and one-way ANOVA. The data were presented
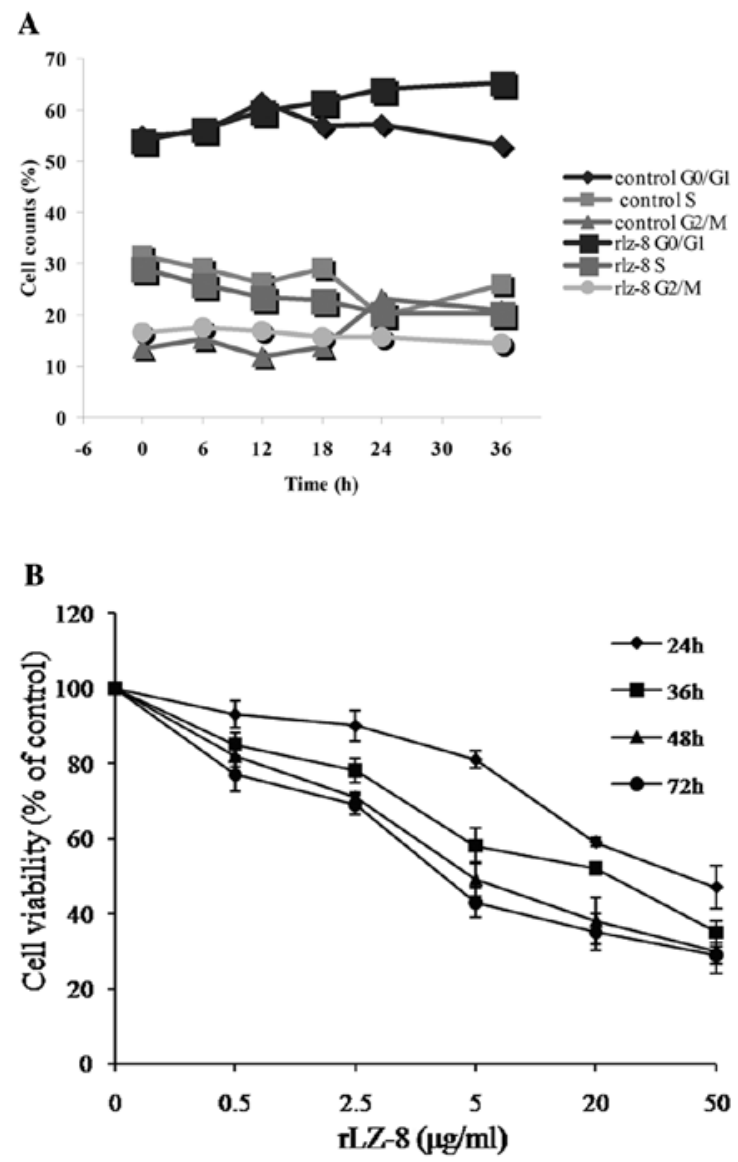

Figure 1. Inhibition of cell growth of SGC7901 cells treated with rLz-8. (A) Effect of rLZ-8 on cell cycle progression in SGC7901. Cells were treated with or without $\mathrm{rLz}-8(5 \mu \mathrm{g} / \mathrm{ml})$ for several time coures $(0,6,12,18,24$ and $36 \mathrm{~h}$ ). Cells were stained with propidium iodide and detected using a flow cytometer. (B) rLz-8 inhibits cell proliferation. SGC7901 cells treated with a range of rLz- 8 concentrations $(0.5$ to $50 \mu \mathrm{g} / \mathrm{ml})$ for $24,36,48$ and $72 \mathrm{~h}$. Proliferation was measured with WST-1 reagent after the indicated period of time. Points represent the mean of three similar experiments $(n=3)$; bars, SE.

as mean \pm standard error (SE). Experiments were performed at least three times. Results were considered to be statistically significant at $\mathrm{P}<0.05$.

\section{Results}

rLz-8 inhibits cell growth and induces cell death in human gastric cancer cell line SGC-7901.rLz-8 caused a detectable accumulation of nuclei in G1 phase with a concomitant decrease of nuclei in $\mathrm{S}$ phase. This was most evident after 12, 18, 24 and $36 \mathrm{~h}$ (Fig. 1A). After 12, 18, 24 and $36 \mathrm{~h}$ of stimulation with $5 \mathrm{mg}$ rLz-8, staining of cells of the gastric cell line SGC-7901 with DNA-specific fluorochrome propidium iodide (PI) showed 59.8, 61.7, 64 and $65.2 \%$ of SGC-7901 cells with G0/G1 DNA content, while $23.3,22.8,20.3$ and $20.4 \%$ of cells were in S-phase, respectively.

Furthermore, treatment of SGC-7901 cells with rLz-8 for $24,36,48$ and $72 \mathrm{~h}$ at different concentrations $(0.5,2.5,5.0,20$, $50 \mu \mathrm{g} / \mathrm{ml}$ ) also resulted in cell growth inhibition. Slight inhibition of proliferation was detected in cells exposed to $0.5 \mu \mathrm{g} /$ $\mathrm{ml}$ of $\mathrm{rLz}-8$, whereas cell proliferation was markedly inhibited in cells treated with 2.5 or $5 \mu \mathrm{g} / \mathrm{ml}$. Treatment with $50 \mu \mathrm{g} / \mathrm{ml}$ rLz- 8 for up to $72 \mathrm{~h}$ significantly reduced the number of viable 
A

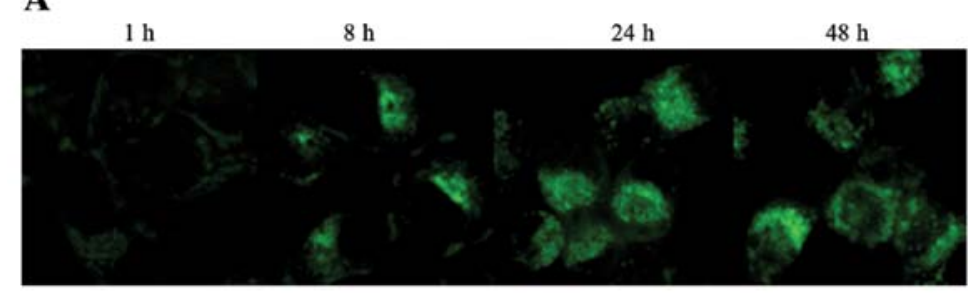

$\mathbf{B}$

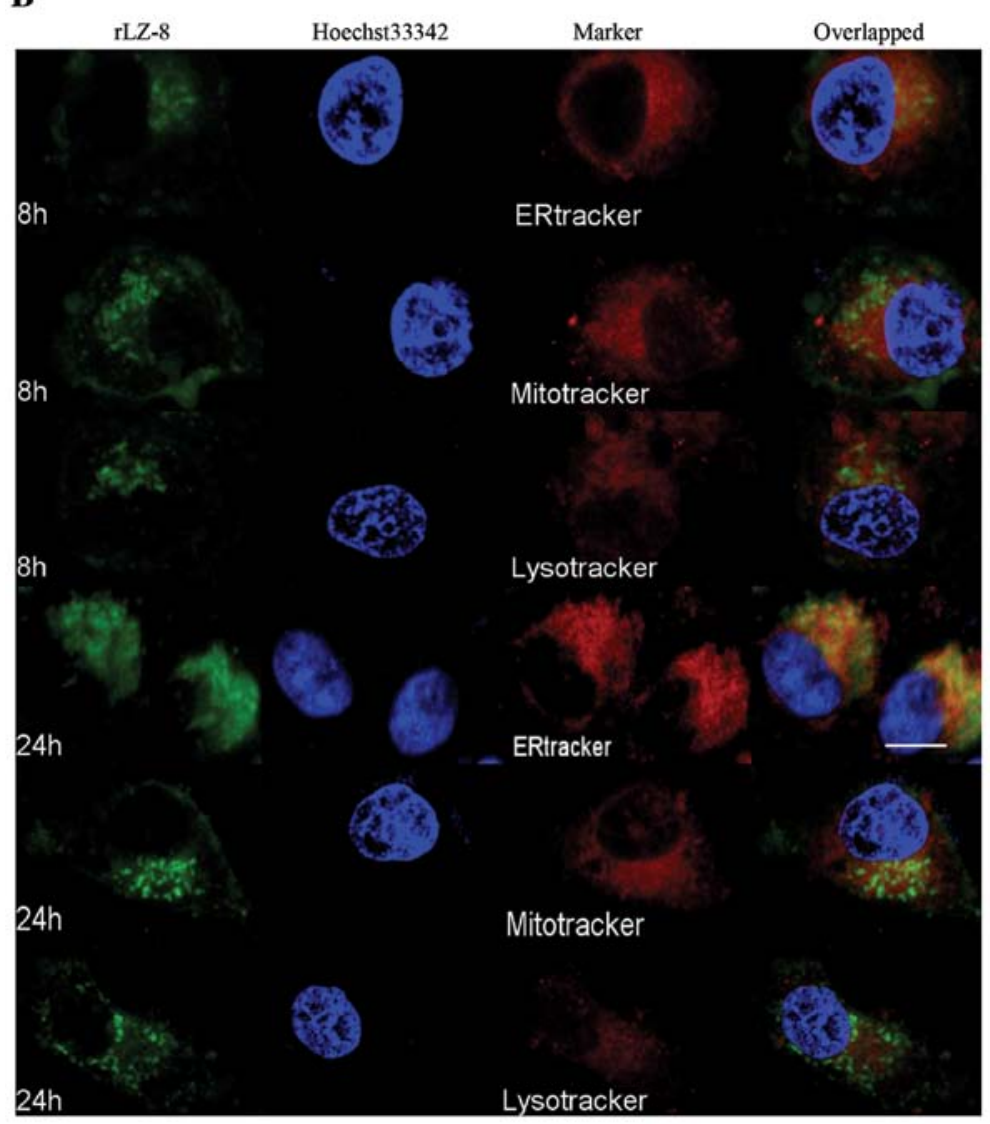

C

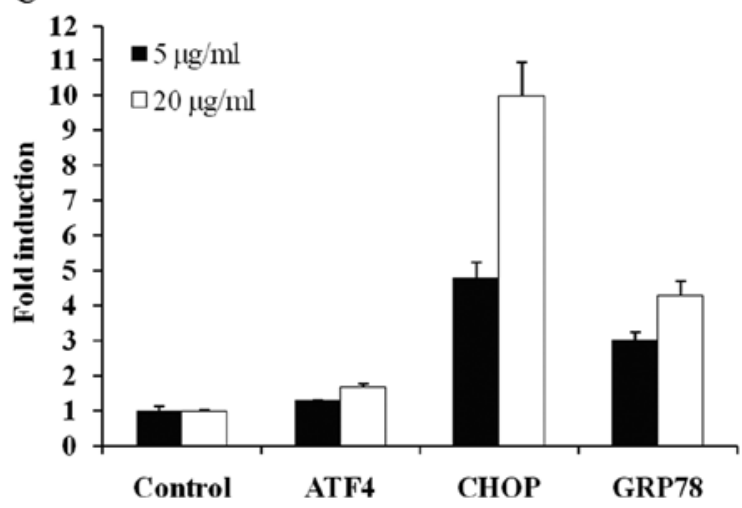

D
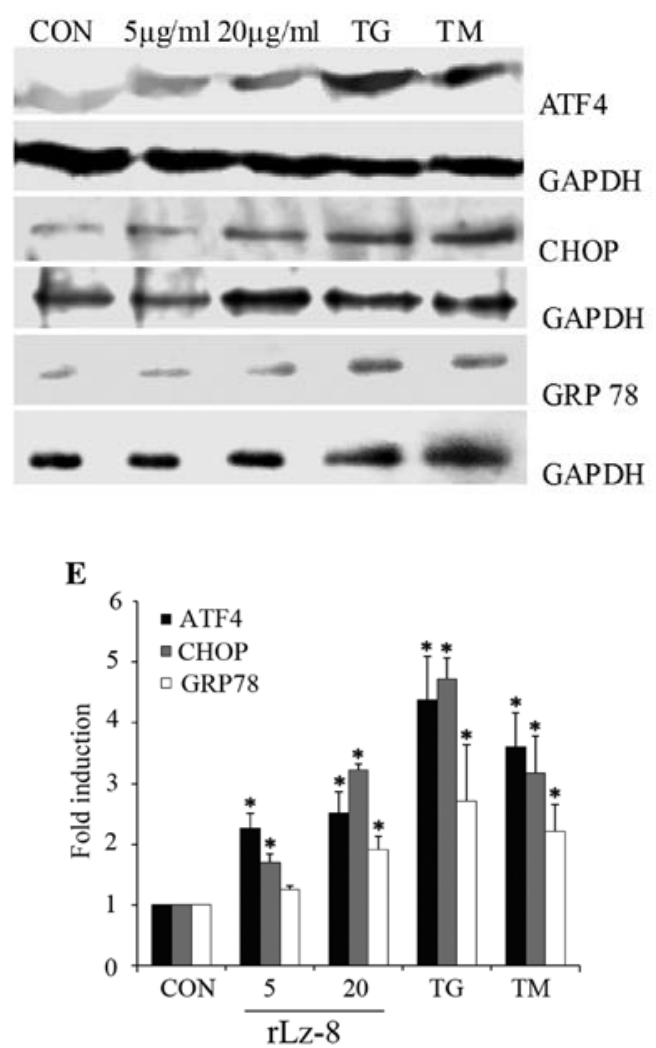

Figure 2. Localization of rLz-8 and induction of ER stress in cells treated with rLz-8. (A) rLz-8- FITC $(5 \mu \mathrm{g} / \mathrm{ml})$ accumulating in cells for several time courses. Scale bar: $10 \mu \mathrm{m}$. (B) Intracellular localization of rLz-8. SGC7901 cells treated with rLz-8-FITC for $24 \mathrm{~h}$. After treatment, the rLz- 8 localization was examined by using Hochest $33342(1 \mu \mathrm{g} / \mathrm{ml})$ for nucleus (blue) and various subcellular makers such as ERtracker (red) for ER, Mitotracker (red) for mitochondria, Lysotracker (red) for lysosome. Scale bar: $10 \mu \mathrm{m}$. (C) Gene expression of ER stress induced by rLz-8. SGC7901 cells treated with rLz-8 (5 or $20 \mu \mathrm{g} / \mathrm{ml} \mathrm{d})$ and incubated for $24 \mathrm{~h}$. Relative mRNA levels were measured by real-time qPCR, n=3. (D) Protein levels of ATF4 (38 kDa), CHOP (30 kDa) and GRP78 (78 kDa) were measured by Western blot analysis. SGC7901 cells were treated for $24 \mathrm{~h}$ with $5 \mu \mathrm{g} / \mathrm{ml} \mathrm{rLz-8,20} \mu \mathrm{g} / \mathrm{ml} \mathrm{rLz}-8,300 \mathrm{nM}$ thapsigargin (TG), and $2 \mu \mathrm{g} / \mathrm{ml}$ tunicamycin (TM). (E) Quantitative analysis of intensities of Western blotting. CON, control. Standard error represents three independent experiments. P-value represents the significant difference between conditions at ${ }^{*} \mathrm{P}<0.05$.

human gastric cancer SGC-7901 cells (Fig. 1B). All results of the cell viability and proliferation assay WST-1 showed that rLz-8 inhibited SGC-7901 cell proliferation in a dose- and time-dependent manner, with $\mathrm{IC}_{50}$ at $24 \mathrm{~h}$ of $5 \mu \mathrm{g} / \mathrm{ml}$.

\section{$r L z-8$ accumulating in nucleus triggers endoplasmic reticulum} stress (ERS)-mediated cell death. The underlying molecular mechanism governing the effects of rLz- 8 on the inhibition of SGC-7901 cell proliferation is still unclear. The first step in examining the possible roles of rLz- 8 was to determine its cellular localization. Colocalization studies were performed using specific markers for the cellular organelles and rLz-8 in SGC-7901 cells. As shown in Fig. 2A and B, fluorescein isothiocyanate (FITC)-labeled rLz-8 and the ER's specific marker overlapped, suggesting that rLz-8 localized at the ER, into which it diffuses.

In the following studies, we examined whether rLz- 8 triggered ER stress as it accumulated in the ER by determining 
A
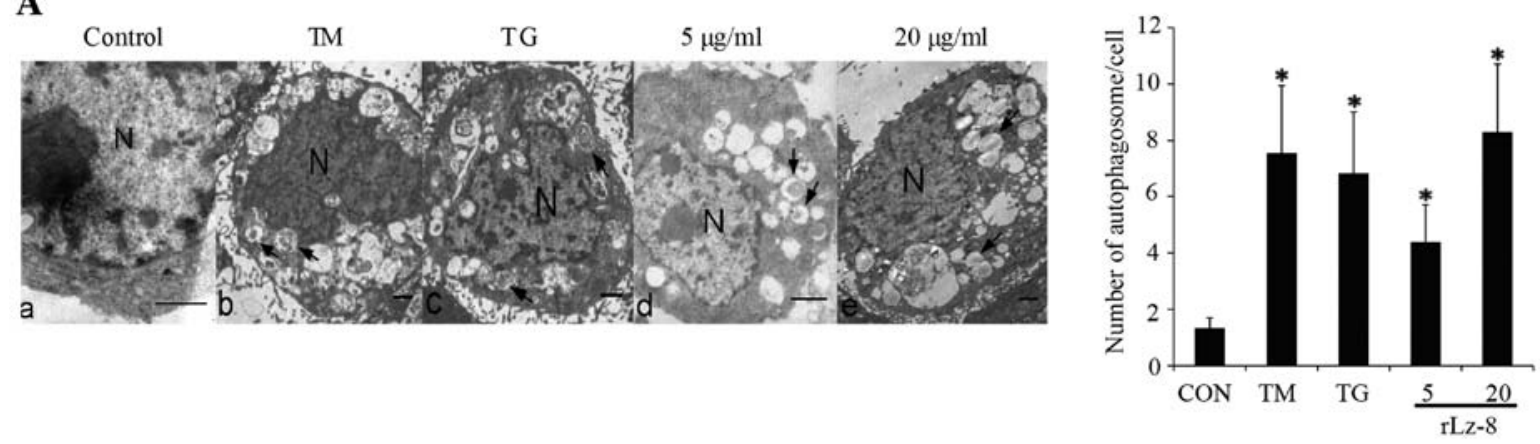

B

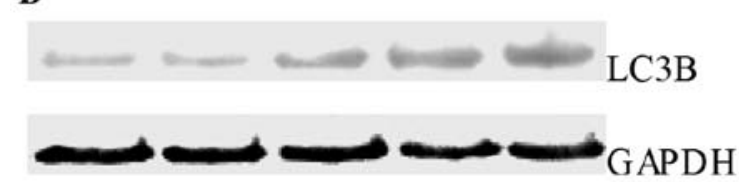

C

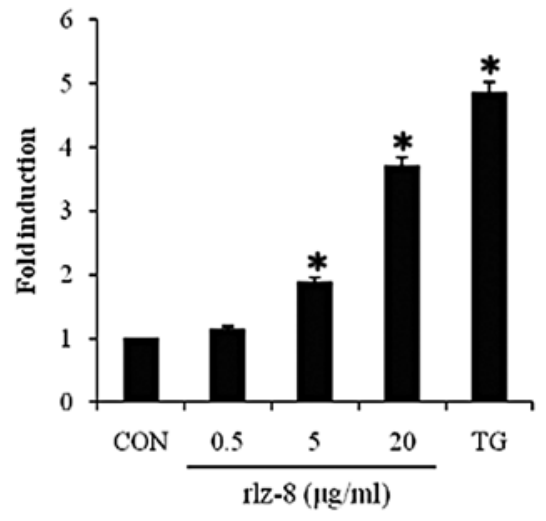

D

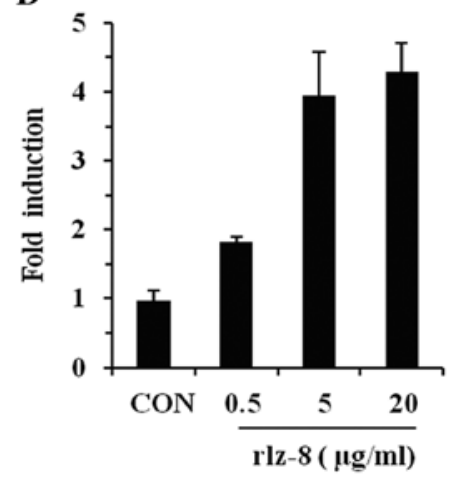

Figure 3A-D. Induction of autophagy in SGC7901 cells treated with rLz-8. (A) Electron micrographs were taken of SGC7901 cells untreated (a) or treated (b) with $2 \mu \mathrm{g} / \mathrm{ml}$ tunicamycin (TM) for $24 \mathrm{~h}$, (c) $300 \mathrm{nM}$ thapsigargin (TG) for $24 \mathrm{~h}$, (d) $5 \mu \mathrm{g} / \mathrm{ml}$ and (e) $20 \mu \mathrm{g} / \mathrm{ml} \mathrm{rLz-}-8$ for $48 \mathrm{~h}$. Double membrane vacuoles are denoted by an arrow, and the nucleus is denoted by N. Scale bar: $1 \mu \mathrm{m}$. The histogram is the numbers of autophagosomes in SGC7901 cells after exposure to the indicated stimuli for $24 \mathrm{~h}$. A total of 25 electron microscopic sections were prepared. Standard error represents 3 independent experiments. P-value represents the significant difference between conditions at " $\mathrm{P}<0.05$. (B) Protein levels of LC3B (15 kDa) were measured by immunoblot analysis. SGC7901 cells were treated for $24 \mathrm{~h}$ with $300 \mathrm{nM}$ thapsigargin (TG), 0.5, 5, and $20 \mu \mathrm{g} / \mathrm{ml} \mathrm{rLz-8.} \mathrm{(C)} \mathrm{Quantitative} \mathrm{analysis} \mathrm{of} \mathrm{intensities} \mathrm{of} \mathrm{Western} \mathrm{blotting.} \mathrm{CON,} \mathrm{control.} \mathrm{Standard}$ error represents 3 independent experiments. P-value represents the significant difference between conditions at " $\mathrm{P}<0.05$. (D) Gene expression of LC3B induced by rLZ-8. SGC7901 cells treated with $0.5,5$, and $20 \mu \mathrm{g} / \mathrm{ml} \mathrm{rLz-8}$ for $24 \mathrm{~h}$. Relative mRNA levels were measured by real-time qPCR, $\mathrm{n}=3$. CON, control.

the effects of rLz-8 on the expression of classical ER stress markers, such as the transcription factors CHOP/GADD153 and ATF4. When ER stress occurs, a large number of unfolded proteins accumulate in the ER, GRP78 dissociates from protein kinase RNA-like ER kinase (PERK) by binding these proteins. Unbound PERK oligomerizes and is activated by trans-autophosphorylation. Activated PERK phosphorylates eIF $2 \alpha$, then its phosphorylation promotes translation of ATF4, escalating ER stress further. ATF4 can function as a homodimer or heterodimer with members of the binding protein family of transcription factors, such as the cell death protein CHOP/ GADD153, and also promotes translation of CHOP/GADD153 $(18,19)$. Real-time quantitative RT-PCR (qRT-PCR) analyses showed that rLz-8 induced CHOP (4- to 9-fold), ATF4 (1- to 2-fold) and GRP78 (3- to 4-fold) mRNA 3- to 10-fold in SGC-7901 cells at 5 and $20 \mu \mathrm{g} / \mathrm{ml}$ (Fig. 2C), and the expression of ATF4 was confirmed by Western blotting (Fig. 2D).
However, in the same test for expression of CHOP, we did not observe positive results. Leena et al (20) reported that some commercially available CHOP antibodies were non-specific for detecting CHOP in Western blotting and hypothesized that antibody specificity may influence the results.

To determine the role of ER stress in the rLz-8-induced death of SGC-7901 cells, we transiently transfected cells with siRNA duplexes specific for CHOP and ATF4. Results showed that CHOP or ATF4 knockdown cells treated with rLz-8 had higher survival rates than the control cells (SCR; Fig. 3E). Therefore, rLz-8 serves as an inducer for death in SGC-7901 cells as it diffuses into the ER and triggers ER stress.

The rLz-8-triggered ER stress induces an autophagic response in SGC-7901 cells. Considering the role of ATF4 in ER stress and autophagy, we next investigated whether rLz-8 induced an 
$\mathbf{E}$
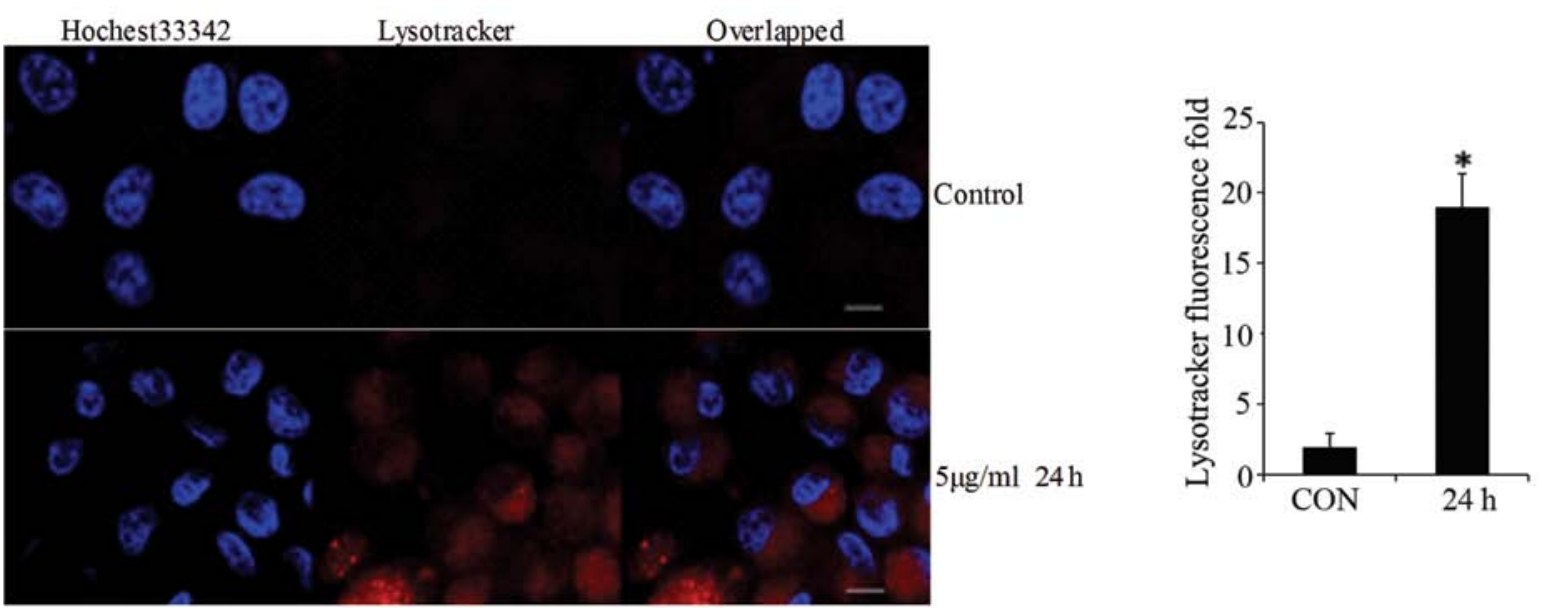

F

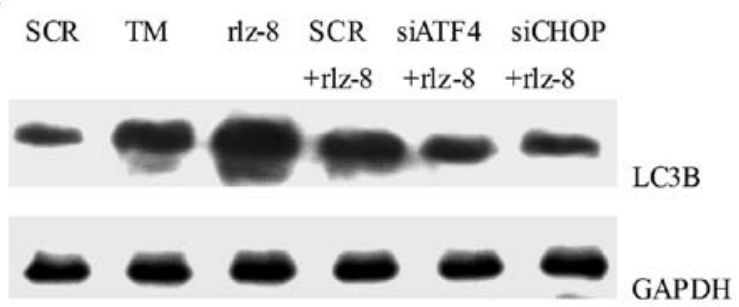

G

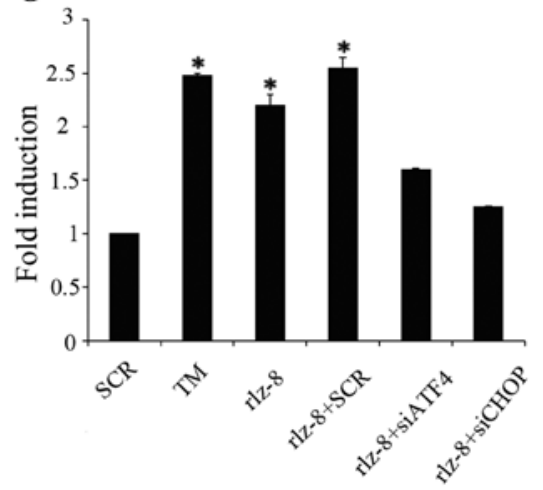

$\mathbf{H}$

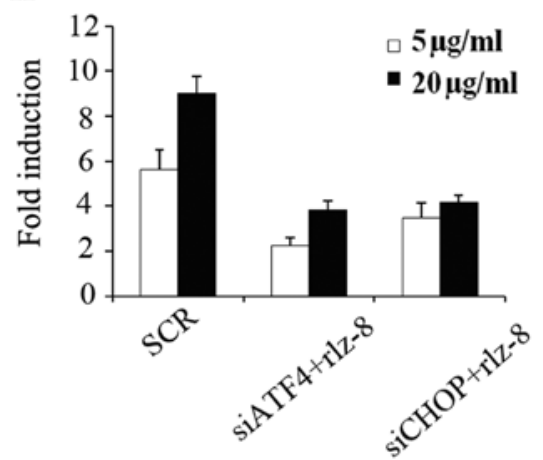

autophagic response in SGC-7901 cells. Electron microscopy remains one of the most accurate methods for the detection of autophagy and quantification of autophagic accumulation. Firstly, autophagic bodies and autophagosomes with double or single membrane structures in the cytoplasm were examined by electron microscopy for autophagic topical changes. In Fig. 3Aa, the electron micrograph depicts the intact cell and nuclear membrane, rough endoplasmic reticulum, distinct nucleus, and homogeneous cytoplasm of normal SGC7901 cells (control). SGC7901 cells treated with different doses of rLz-8 are shown in Fig. 3Ad and e. Membrane-bound autophagosomes in the cytoplasm are observed. The nucleus is condensed, but the nuclear membrane is intact and distinct. Tunicamycin (TM) and thapsigargin (TG) are triggers of the autophagic ER stress-induced response in cells. As shown in the electron micrograph in Fig. 3Ab and c, SGC7901 cells treated with TM and TG show an abnormally large increase
Figure 3E-H. Induction of autophagy in SGC7901 cells treated with rLz-8. (E) Induction of lysosomes in SGC7901 cells treated with rLz-8. SGC7901 cells were treated with $5 \mu \mathrm{g} / \mathrm{ml}$ of rLz- 8 for $24 \mathrm{~h}$. Nuclear stained with Hoechst 33342 (blue) and Lysotracker (red) staining are shown. The histogram is quantitative analysis of fluorescence intensities. Scale bar: $10 \mu \mathrm{m}$. (F) Protein levels of LC3B (15 kDa) were measured by immunoblot analysis. Cells were RNAi against control (SCR), ATF4 and CHOP. After $24 \mathrm{~h}$, cells were treated with $5 \mu \mathrm{g} / \mathrm{ml} \mathrm{rLz}-8$ for $24 \mathrm{~h}$. (G) Quantitative analysis of intensities of Western blotting. $\mathrm{CON}$, control. Standard error represents three independent experiments. $\mathrm{P}$-value represents the significant difference between conditions at ${ }^{*} \mathrm{P}<0.05$. (H) Gene expression of LC3B with siRNA. Cells were RNAi against control (SCR), ATF4 and CHOP. Cells were treated $24 \mathrm{~h}$ later with $5 \mu \mathrm{g} / \mathrm{ml}$ or $20 \mu \mathrm{g} /$ $\mathrm{ml} \mathrm{rLz}-8$ for $24 \mathrm{~h}$. Relative mRNA levels were measured by real-time qPCR, $\mathrm{n}=3$.

in the number of autophagosomes, condensed nuclei, and damaged nuclear membranes. The number of autophagosomes was significantly increased in cells treated with these stimuli, compared to non-treated cells (Fig. 1B).

Microtubule-associated protein 1 light chain 3 (MAP1-LC3) is essential for autophagy and is associated with autophagosome membranes. MAP1-LC3 has two subunits, specifically MAP1-LC3A (MAP1-LC3 $\alpha$ ) and MAP1-LC3B (MAP1-LC3 $\beta$ ) (21). There are two forms of LC3B, the cytosolic LC3-I and the membrane-bound LC3-II. During autophagy, LC3B is synthesized in large quantities. LC3-I is formed by the removal of the C-terminal 22 amino acids from newly synthesized LC3B, and a fraction of LC3-I is converted into LC3-II $(22,23)$. Therefore, LC3B is thought to be an important marker for autophagy. During rLz- 8 treatment, the protein and mRNA levels of LC3B increased significantly in a dose- and time-dependent manner (Fig. 3B and C), and there was an increase in the processed form 
A

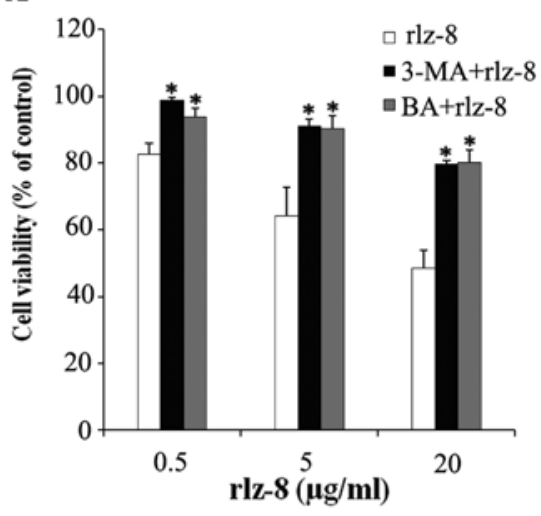

B
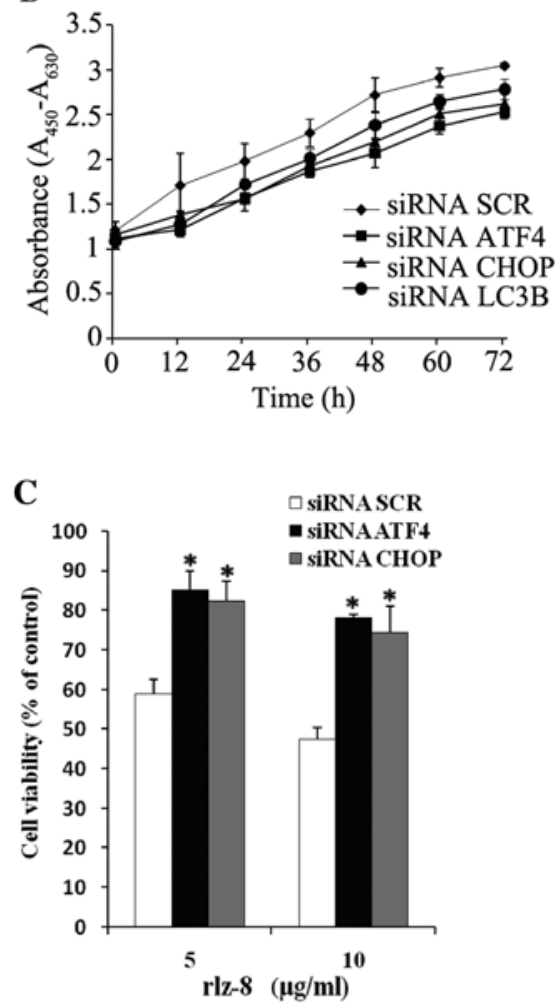

D

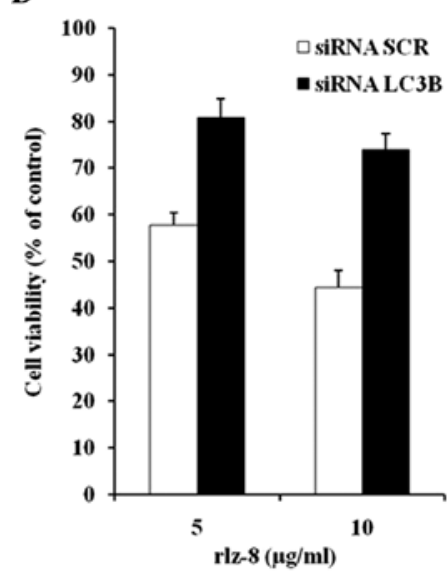

of LC3B, which was indicative of increased autophagy. The result of real-time reverse-transcription PCR analysis showed that for the negative control (CON), positive control (TG) and

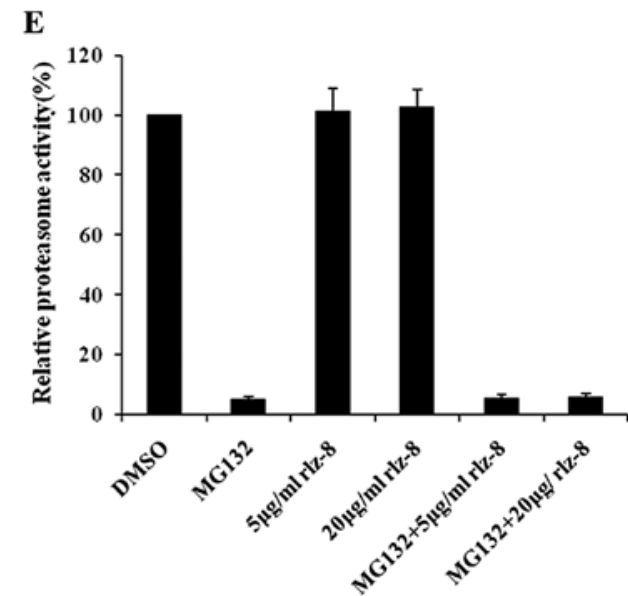

Figure 4. The role of autophagy in the death of SGC7901 cells treated with rLz-8. (A) Effect of rLz-8-induced autophagic cell death. SGC7901 cells non-treated or treated with $1 \mathrm{mM} 3-\mathrm{MA}$ or $30 \mu \mathrm{M}$ BA for $1 \mathrm{~h}$, and then treated with $0.5,5$ or $20 \mu \mathrm{g} / \mathrm{ml} \mathrm{rLz}-8$ for $24 \mathrm{~h}$. Proliferation was measured with WST-1 reagent after the indicated time period. Standard error represents 3 independent experiments. P-value represents the significant difference between conditions at ${ }^{*} \mathrm{P}<0.05$. (B) Measurement of proliferation of SGC7901 cells in response to siRNA. Cells were transfected with siRNA against control (SCR), ATF4, and CHOP and siRNA LC3B. Six hours later, cells were cultured with DMEM supplemented with $10 \%$ FBS and WST-1 reagent was added after the indicated period of time. The absorbance was determined at the respective wavelength with an ELISA reader. Standard error represents 4 independent experiments. (C and D) Cell viability of SGC7901 cells treated with rLz-8. Cells were transfected with siRNA against control (SCR), ATF4, CHOP and siRNA LC3B. (E) Proteasome activity in SGC7901 cells treated with rLz-8. Proteasome activity in cells was directly measured using a cellbased assay after cells were treated with different combinations of drugs for $24 \mathrm{~h}$. MG132 $(100 \mathrm{nM})$ is a proteasome inhibitor. Standard error represents three independent experiments.

rLz-8-treated groups $(0.5,5$ and $20 \mu \mathrm{g} / \mathrm{ml})$, mRNA levels of LC3B were up-regulated 1.8-, 3.9-, and 4.2-fold, respectively.

During rLz- 8 treatment, the protein and mRNA levels of LC3, a recognized marker, increased significantly in a dose- and time-dependent manner (Fig. 3D), and there was an increase in the processed form of LC3. This was indicative of increased autophagy, particularly with 5 and $20 \mu \mathrm{g} / \mathrm{ml}$, between the negative control (CON), positive control (TM, TG) and rLz-8-treated groups.

When autophagy is induced in old or unwanted organelles, proteins and microbes are delivered to the lysosome, accompanied by a rapid increase in the number of lysosomes. Lysotracker, a lysosome-specific dye, was used to examine the number of lysosomes in rLz-8-treated SGC-7901 cells. Results indicated that compared to the negative control group, cells treated with $\mathrm{rLz}-8$ at $5 \mu \mathrm{g} / \mathrm{ml}$ for $24 \mathrm{~h}$ demonstrated a significant increase in lysosomal mass (Fig. 3E).

The relationship between autophagy and ER-stress was investigated by using siRNA duplexes specific for the ER-stress markers, CHOP and ATF. When CHOP and ATF4 were rendered dysfunctional, the LC3B protein and mRNA levels were greatly reduced compared to the control siRNAtransfected cells (Fig. 3F-H). Hence, we suggest that ER-stress is the main factor triggering the autophagy response.

The role of autophagy in the death of SGC7901 cells treated with $r L z-8$. Over the years many studies have proven that 


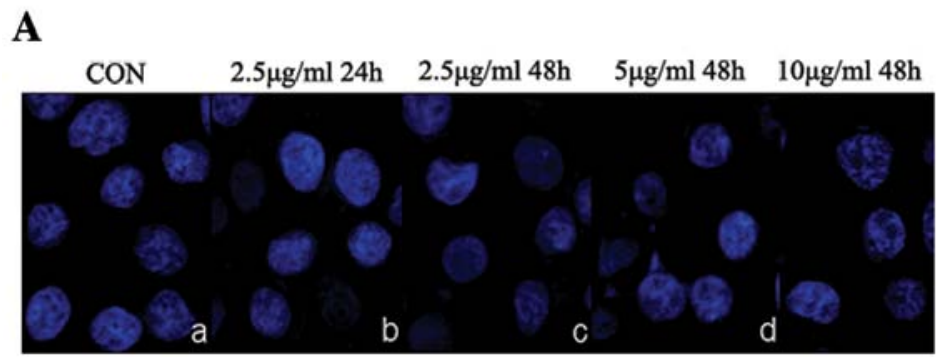

$\mathbf{B}$
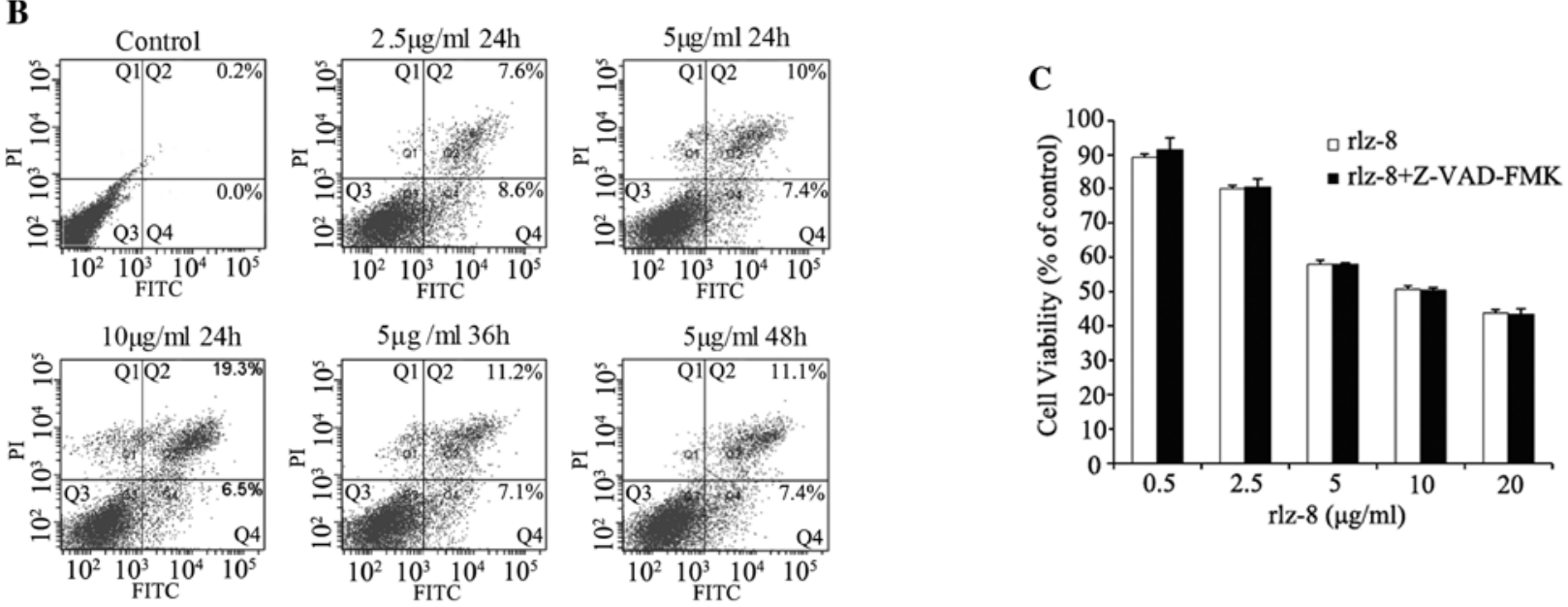

D
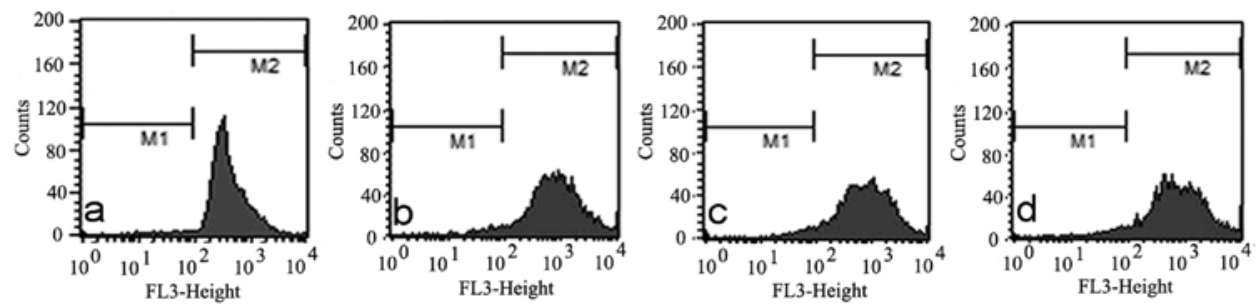

Figure 5. (A) Morphological changes of nuclear in SGC7901 cells treated with rLz-8. SGC7901 cells untreated (a) or treated (b-e) with $2.5,5$, or $10 \mu \mathrm{g} / \mathrm{ml}$ rLz-8 for several time courses ( $24 \mathrm{~h}, 48 \mathrm{~h}$ ), and then $1 \mu \mathrm{g} / \mathrm{ml}$ Hoechst 33342 stained nuclear for $20 \mathrm{~min}$. (B) Effect of rLz- 8 on cell viability of SGC7901 cells. AV/PI double staining was performed on SGC7901 cells before and exposure to 2.5, 5, and $10 \mu \mathrm{g} / \mathrm{ml} \mathrm{rLz}-8$ for different periods of time (24,36 and $48 \mathrm{~h})$. AV- $/$ $\mathrm{PI}^{+}(\mathrm{Q} 1)$ : percentage early necrotic cells; $\mathrm{AV}^{+} / \mathrm{PI}^{+}(\mathrm{Q} 2)$ : percentage late apoptotic and dead cells, $\mathrm{AV} / \mathrm{PI}-(\mathrm{Q} 3)$ : percentage viable cells, $\mathrm{AV}^{+} / \mathrm{PI}^{-}\left(\mathrm{Q}^{-}\right)$: percentage early apoptotic cells. (C) Effect of caspases in SGC7901 cells treated with rLz-8. SGC7901 cells untreated or treated with $50 \mu \mathrm{m} \mathrm{Z-VAD-FMK} \mathrm{for} 30$ min, and then treated with 0.5 to $20 \mu \mathrm{g} / \mathrm{ml} \mathrm{rLz}-8$ for $48 \mathrm{~h}$. The result measured with WST1. Standard error represents 4 independent experiments. (D) Effect of rLz- 8 on mitochondrial membrane potential of SGC7901 cells. SGC7901 cells untreated (a) or treated (b-d) with $0.5,5$, and $20 \mu \mathrm{g} / \mathrm{ml} \mathrm{rLz}-8$ for $24 \mathrm{~h}$, and then cells were incubated with $10 \mu \mathrm{g} / \mathrm{ml} \mathrm{JC}-1$ for $30 \mathrm{~min}$.

autophagy plays a dual role in the biological process of cell death. To examine whether the autophagy induced by rLz-8 plays any roles in cell survival or death, cells in which autophagy was blocked by 3-methyladenine (3-MA; a specific inhibitor of autophagic/lysosomal protein degradation) were treated with rLz-8. A colorimetric WST-1 assay was used to determine the number of viable cells. Results showed that cell death that would otherwise be induced by rLz- 8 was inhibited: the number of rLz-8-treated living cells was more than the control that was not treated with 3-MA (Fig. 4A). We next transiently transfected cells with siRNA duplexes specific for LC3B, then detected the survival rate of LC3B knockdown cells treated with rLz- 8 for 24 or $48 \mathrm{~h}$. Compared with the non-siRNA LC3B control in which expression of LC3B was inhibited, the rLz-8 killing effect declined rapidly (Fig. 4D). Hence, this suggests that autophagy plays a crucial role in the process of cell death induced by rLz-8.

Caspase inhibitors do not prevent $r L z-8$-induced cell death. When apoptosis occurs, significant morphological changes such as chromatin condensation, nuclear shrinkage, and the disappearance of the nuclear envelope and nucleolus may be seen in cells. To investigate the morphological changes of organelles in rLz8-treated SGC-7901 cells, laser scanning confocal microscopy was used to analyze Hochest 33342-stained nuclei. As shown in Fig. 2A, normal cells have a beam-shaped nucleus, smooth nuclear envelope, and non-condensed zones of chromatin. The cancer cell line SGC-7901 was treated with rLz- 8 for 24 and $48 \mathrm{~h}$ 
at concentrations ranging from 2.5 to $10 \mu \mathrm{g} / \mathrm{ml}$. Compared with normal cells, no apoptotic morphological changes were seen in any of the rLz-8-treated groups (Fig. 2A). Most significantly, in the high-dose rLz- 8 treatment group $(10 \mu \mathrm{g} / \mathrm{ml})$, nearly all cells were dead, but we did not observe, by transmission electron microscopy (TEM), any apoptotic morphological changes that had occurred during the process of death (Fig. 3A). We also analyzed the apoptosis rate of the treatment groups by using flow cytometry (Fig. 5B), but results indicated that $\mathrm{rLz}-8$ could not induce apoptosis in SGC-7901 cancer cells in a time- and dose-dependent manner.

Although it is accepted that apoptosis is mediated by caspase activity, the specific role of caspases is debatable (17,24-29). A caspase inhibition assay was performed to examine the involvement of caspase in rLz-8-induced cell death. The caspase inhibitor z-VAD-fmk (50 mM) did not significantly affect the cell viability of rLz-8-treated SGC-7901 cells (Fig. 5C). These results proved that $\mathrm{rLz}-8$-induced cell death is independent of caspase activation. It is well known that when apoptosis occurs, a change in the electric potential of the mitochondrial membrane is a typical event. Herein, flow cytometry was used to investigate changes in mitochondrial membrane potential in rLz-8-treated SGC7901 cells. These data together with the siRNA assay results corroborate that rLz-8-induced cell death occurs by autophagy.

\section{Discussion}

Disturbances in normal functions of the ER lead to the UPR, an evolutionarily conserved cell stress response which is aimed initially at compensating for damage but can eventually trigger cell death if ER dysfunction is severe or prolonged. The mechanisms by which ER stress leads to cell death remain enigmatic, with multiple potential participants described with little clarity, with specific death effectors dominating in particular cellular contexts (30).

As we know, types of cell death induced by ER stress include apoptotic cell death, necrosis, and autophagic cell death (31). Among these, apoptotic cell death depends greatly on caspase activity. Many important relevant mechanisms involved in caspase-dependent cell death have already been studied extensively (32).

In our study, rLz-8 exerted a promising killing effect on SGC-7901 cells. We used classical methods to examine whether caspase-independent cell death rather than caspasedependent apoptosis was triggered by rLz-8-induced ER stress. The results of TEM indicated that no morphological feature of caspase-dependent apoptosis was observed in rLz-8-treated cells, i.e., chromatin condensation, loss of cytoplasmic processes, round cell shape or fading of the nuclear membrane (33-36). Moreover, when caspase families were inhibited with the pan-caspase inhibitor z-VAD-fmk in SGC-7901 cells, we found that rLz- 8 still had the same killing effect, affirming that rLz-8-induced cell death is not caspase-dependent, and may not even be apoptosis (37-41).

Regarding rLz-8-induced cell death, our findings suggest: 1) In SGC-7901 cells, rLz-8 diffuses and accumulates gradually in the ER, reaching saturation after $24 \mathrm{~h}$. rLz-8 was not transported out of the ER or degraded until the cells died. 2) After rLz-8 accumulated in the ER, a significant increase in the number of lysosomes was observed, by fluorescence microscopy. The results of TEM also confirmed dilated and vacuolized smooth ER and an increased number of lysosomes. 3) rLz-8-treated cells showed increased mRNA and protein levels of $\mathrm{CHOP} /$ GADD153 and ATF4, respectively. 4) When rLz-8 gradually accumulated in the ER of SGC-7901 cells, autophagy and cell death were observed. When autophagy was inhibited by 3-MA treatment, the number of dead cells decreased, suggesting that autophagy played a role in promoting cell death in $\mathrm{Lz}-8$ treated cells.

Therefore, how does the cell deal with the aggregation of rLz-8 in the ER? Excess amounts of unfolded or misfolded proteins in the ER are retrotranslocated to the cytoplasm and degraded by the ERAD system, which includes a ubiquitin/ proteasome and an autophagy/lysosome system (15). A larger number of autophagosomes and lysosomes appeared in rLz8-treated cells. This suggests that the autophagy/lysosome system was activated, and the cell may employ this system to degrade rLz-8. In the study of Fujita et al (5), native and mutant dysferlin were degraded by the ubiquitin/proteasome ERAD system. However, when mutant dysferlin was not sufficiently degraded by ubiquitin/proteasome ERAD system, it aggregated in the ER and stimulated autophagy formation and LC3 conversion via the ER stress-related PERK-eIF2a-ATF4-CHOP/GADD153 pathway. This pathway was also activated in rLz-8-treated cells. The mutant dysferlin aggregates on the ER membrane could be recognized by the elongating membrane that forms the autophagosome, and is then degraded by the autolysosome upon fusion with a lysosome. Although there were no experimental results in our study verifying that rLz-8 was recognized and degraded by the ubiquitin/proteasome ERAD system, we used a Bayesian discriminant method $(42,43)$ to predict the rLz-8 ubiquitination sites. However, specific ubiquitination sites were not found.

In general, the ERAD system degrades aberrant proteins to ensure cell survival, but in this study the SGC-7901 cells were killed by the accumulation of $\mathrm{rLz}-8$ in the ER. This is similar to the apoptotic death of $\mathrm{C} 2 \mathrm{C} 5$ cells caused by dysferlin aggregates, or protein overexpressing in yeast. It is known that excessive ER stress, or ER-stress mediated cell death, can kill cells but what are the roles of ERAD and autophagy in this process? According to a recent study, autophagy formation was a cellular defense mechanism against polyQ72-induced ER-stress-mediated cell death, whose mechanism involved the degradation of polyQ72 aggregates (44). Similar studies also indicated that autophagy protects cells from ER stress arising from protein aggregations via the ERAD system $(45,46)$. However, we have determined that while aggregations of rLz-8 induced ER stress and triggered the ERAD system, the resulting autophagy contributed to cell death rather than survival. Before this, it was very rare that a heterologous protein aggregating in the ER of a tumor cell could trigger autophagy-dependent cell death.

Next, what role did ER stress play in the rLz-8-induced cell death? As we know, when ER stress exceeds a threshold it mediates many types of cell death, including classic apoptosis, autophagic cell death and necrosis. The principal challenge with any cellular strategy for preventing death caused by ER stress lies with the multitude of parallel pathways that potentially lead to downstream cell death mechanisms. Multiple pathways 
linking ER stress to cell death have been reported. Of these, ATF4-CHOP/GADD153 is the first identified protein that mediates ER stress-induced apoptosis and much is known of the roles of this molecule in cell death. Nevertheless, little is known of just how ATF4-CHOP/GADD153 induces cell death, especially considering that CHOP-deficient cells are resistant to ER stress-mediated cell death. Our study showed that when expression of ATF4 or CHOP was inhibited in rLz-8-treated SGC-7901 cells, dead cells decreased significantly, and LC-3II conversion was down-regulated. This proves that the ATF4-CHOP pathway and ER-stress both are the key factors for inducing cell death, and also that the ATF4-CHOP pathway acts as a bridge between ER stress and autophagy.

In conclusion, rLz-8 aggregation induces autophagic cell death and provides a novel mechanism for protein-based anticancer medicine. This may lead to new strategies to develop therapeutic drugs that will target cancer cells to undergo autophagic cell death independent of apoptosis.

\section{References}

1. Parodi AJ: Protein glucosylation and its role in protein folding. Annu Rev Biochem 69: 69-93, 2000.

2. Lai E, Teodoro T and Volchuk A: Endoplasmic reticulum stress: signaling the unfolded protein response. Physiol (Bethesda) 22: 193-201,2007.

3. Boelens J, Lust S, Offner F, Bracke ME and Vanhoecke BW: The endoplasmic reticulum: a target for new anticancer drugs. In Vivo 21: 215-226. 2007.

4. McGuckin MA, Eri RD, Das I, Lourie R and Florin TH: ER stress and the unfolded protein response in intestinal inflammation. Am J Physiol Gastrointest Liver Physiol 298: G820-G832, 2010.

5. Fujita E, Kouroku Y, Isoai A, Kumagai H, Misutani A, Matsuda C Hayashi YK and Momoi T: Two endoplasmic reticulum-associated degradation (ERAD) systems for the novel variant of the mutant dysferlin: ubiquitin/proteasome ERAD(I) and autophagy/ lysosome ERAD(II). Hum Mol Genet 16: 618-629, 2007.

6. Ohsumi Y: Molecular dissection of autophagy: two ubiquitin-like systems. Nat Rev Mol Cell Biol 2: 211-216, 2001.

7. Komatsu M, Waguri S, Ueno T, Iwata J, Murata S, Tanida I, Ezaki J, Mizushima N, Ohsumi Y and Uchiyama Y: Impairment of starvation-induced and constitutive autophagy in Atg7deficient mice. J Cell Biol 169: 425-434, 2005.

8. Chen Y, McMillan-Ward E, Kong J, Israels SJ and Gibson SB: Oxidative stress induces autophagic cell death independent of apoptosis in transformed and cancer cell. Cell Death Differ 15: 171-182, 2008

9. Kopito RR and Ron D: Conformational disease. Nat Cell Biol 2 E207-E209, 2000.

10. Miyazaki T and Nishijima M: Studies on fungal polysaccharides XXVII. Structural examination of a water-soluble, antitumor polysaccharide of Ganoderma lucidum. Chem Pharm Bull (Tokyo) 29: 3611-3616, 1981

11. Wang SY, Hsu ML, Hsu HC, Tzeng CH, Lee SS, Shiao MS and Ho CK: The anti-tumor effect of Ganoderma lucidum is mediated by cytokines released from activated macrophages and T lymphocytes. Int J Cancer 70: 699-705, 1997.

12. Nonaka Y, Shibata H, Nakai M, Kurihara H, Ishibashi H, Kiso Y, Tanaka T, Yamaguchi $\mathrm{H}$ and Abe S: Anti-tumor activities of the antlered form of Ganoderma lucidum in allogeneic and syngeneic tumor-bearing mice. Biosci Biotechnol Biochem 70: 2028-2034, 2006.

13. Kino K, Yamashita A, Yamaoka K, Watanabe J, Tanaka S, Ko K, Shimizu K and Tsunoo H: Isolation and characterization of a new immunomodulatory protein, ling zhi-8 (LZ-8), from Ganoderma lucidum. J Biol Chem 264: 472-478, 1989.

14. Tanaka S, Ko K, Kino K, Tsuchiya K, Yamashita A, Murasugi A, Sakuma S and Tsunoo H: Complete amino acid sequence of an immunomodulatory protein, Ling Zhi-8 (Lz-8). An immunomodulator from a fungus, Ganoderma lucidum, having similarity to immunoglobulin variable regions. J Biol Chem 264: 16372-16377, 1989.
15. Huang L, Sun F, Liang C, He YX, Bao R, Liu L and Zhou CZ: Crystal structure of LZ-8 from the medicinal fungus Ganoderma lucidum. Proteins 75: 524-527, 2009.

16. Liao CH, Hsiao YM, Sheu GT, Chang JT, Wang PH, Wu MF, Wu MF, Shieh GJ, Hsu CP and Ko JL: Nuclear translocation of telomerase reverse transcriptase and calcium signaling in repression of telomerase activity in human lung cancer cells by fungal immunomodulatory protein from Ganoderma tsugae. Biochem Pharmacol 74: 1541-1554, 2007.

17. Momoi T: Caspases involved in ER stress-mediated cell death. J Chem Neuroanat 28: 101-105, 2004.

18. Harding H, Zhang Y and Ron D: Translation and protein folding are coupled by an endoplasmic reticulum resident kinase. Nature 397: 271-274, 1999.

19. Rzymski T and Harris AL: The unfolded protein response and integrated stress response to anoxia. Clin Cancer Res 13: 2537-2540, 2007.

20. Leena H, Tatyana G, Huang CJ and Butler PC: Many commercially available antibodies for detection of $\mathrm{CHOP}$ expression as a marker of endoplasmic reticulum stress fail specificity evaluation. Cell Biochem Biophys 51: 105-107, 2008.

21. Mann SS and Hammarback JA: Gene localization and developmental expression of light chain 3: a common subunit of microtubule-associated protein 1A (MAP1A) and MAP1B. J Neurosci Res 43: 535-544, 1996.

22. Kabeya Y, Mizushima N, Ueno T, Yamamoto A, Kirisako T, Noda T, Kominami E, Ohsumi Y and Yoshimori T: LC3, a mammalian homolog of yeast Apg8p, is localized in autophagosome membrane after processing. EMBO J 19: 5720-5728, 2000 .

23. Shintani T and Klionsky DJ: Autophagy in health and disease: a double-edged sword. Science 306: 990-995, 2004.

24. Yoneda T, Imaizumi K, Oono K, Yui D, Gomi F, Katayama T and Tohyama M: Activation of caspase-12, an endoplastic reticulum (ER) resident caspase, through tumor necrosis factor receptorassociated factor 2-dependent mechanism in response to the ER stress. J Biol Chem 276: 13935-13940, 2001.

25. Fischer H, Koenig U, Eckhart L and Tschachler E: Human caspase 12 has acquired deleterious mutations. Biochem Biophys Res Comm 293: 722-726, 2002.

26. Szegezdi E, Fitzgerald U and Samali A: Caspase-12 and ER-stress-mediated apoptosis: the story so far. Ann NY Acad Sci 1010: 186-194, 2003.

27. Hitomi J, Katayama T, Eguchi Y, Kudo T, Taniguchi M, Koyama Y, Manabe T, Yamagishi S, Bando Y, Imaizumi K, Tsujimoto $\mathrm{Y}$ and Tohyama $\mathrm{M}$ : Involvement of caspase-4 in endoplasmic reticulum stress-induced apoptosis and $\mathrm{A} \beta$-induced J Cell Biol 165: 347-356, 2004.

28. Martinez JA, Zhang Z, Svetlov SI, Hayes RL, Wang KK and Larner SF: Calpain and caspase processing of caspase-12 contribute to the ER stress-induced cell death pathway in differentiated PC12 cells. Apoptosis 15: 1480-1493, 2010.

29. Salvesen GS: Caspases and apoptosis. Essays Biochem 38: 9-19, 2002.

30. Kim I, Xu W and Reed JC: Cell death and endoplasmic reticulum stress: disease relevance and therapeutic opportunities. Nat Rev Drug Discov 7: 1013-1030, 2008.

31. Bursch W, Ellinger A, Gerner C, Fröhwein U and SchulteHermann R: Programmed cell death (PCD). Apoptosis, autophagic PCD, or others? Ann NY Acad Sci 926: 1-12, 2000.

32. Kim R, Emi M and Tanabe K: Caspase-dependent and -independent cell death pathways after DNA damage. Oncol Rep 14: 595-599, 2005.

33. Stuppia L, Gobbi P, Zamai L, Palka G, Vitale M and Falcieri E: Morphometric and functional study of apoptotic cell chromatin. Cell Death Differ 3: 397-405, 1996.

34. Doonan F and Cotter TG: Morphological assessment of apoptosis. Methods 44: 200-204, 2008.

35. Lin JC, Ho YS, Lee JJ, Liu CL, Yang TL and Wu CH: Induction of apoptosis and cell-cycle arrest in human colon cancer cells by meclizine. Food Chem Toxicol 45: 935-944, 2007.

36. Moodley YP,Caterina P, Scaffidi AK, Misso NL,Papadimitriou JM, McAnulty RJ, Laurent GJ, Thompson PJ and Knight DA: Comparison of the morphological and biochemical changes in normal human lung fibroblasts and fibroblasts derived from lungs of patients with idiopathic pulmonary fibrosis during FasL-induced apoptosis. J Pathol 202: 486-495, 2004.

37. Nicotera P, Leist M, Single B and Volbracht C: Execution of apoptosis: converging or diverging pathways? Biol Chem 380: 1035-1040, 1999 . 
38. Nicotera P, Leist M, Fava E, Berliocchi L and Volbracht C: Energy requirement for caspase activation and neuronal cell death. Brain Pathol 10: 276-282, 2000.

39. Cahir-McFarland $E$ and Kieff E: NF-kappaB inhibition in EBV-transformed lymphoblastoid cell lines. Recent Results Cancer Res 159: 44-48, 2002.

40. Graczyk PP: Caspase inhibitors as anti-inflammatory and antiapoptotic agents. Prog Med Chem 39: 1-72, 2002.

41. Masuoka HC, Guicciardi ME and Gores GJ: Caspase inhibitors for the treatment of hepatitis C. Clin Liver Dis 13: 467-475, 2009.

42. Tung CW and Ho SY: Computational identification of ubiquitylation sites from protein sequences. BMC Bioinformatics 9: 310, 2008.

43. Xue Y, Chen H, Jin C, Sun Z and Yao X: NBA-Palm: prediction of palmitoylation site implemented in Naive Bayes algorithm. BMC Bioinformatics 7: 458, 2006.
44. Kouroku Y, Fujita E, Tanida I, Ueno T, Isoai A, Kumagai H, Ogawa S, Kaufman RJ, Kominami E and Momoi T: ER stress (PERK/eIF2a phosphorylation) mediates the polyglutamineinduced LC3 conversion, an essential step for autophagy formation. Cell Death Differ 14: 230-239, 2007.

45. Ravikumar B, Duden R and Rubinsztein DC: Aggregate-prone proteins with polyglutamine and polyalanine expansions are degraded by autophagy. Hum Mol Genet 11: 1107-1117, 2002.

46. Ravikumar B, Vacher C, Berger Z, Davies JE, Luo S, Oroz LG, Scaravilli F, Easton DF, Duden R, O'Kane CJ and Rubinsztein DC: Inhibition of mTOR induces autophagy and reduces toxicity of polyglutamine expansions in fly and mouse models of Huntington disease. Nat Genet 36: 585-595, 2004. 